\title{
A Generative Approach to the Modeling of Isomorphic Hidden-Figure Items
}

\author{
Isaac I. Bejar \\ Educational Testing Service \\ Peter Yocom \\ Rensselaer Polytechnic Institute
}

\begin{abstract}
A generative approach to psychometric modeling consists of encoding information about the cognitive processes and structures that underlie test performance into an item-generation algorithm in such a way that the generated items. have known psychometric parameters. An important by-product of the approach is that the knowledge about the response process is tested every time a test is administered. Validation thus becomes an ongoing process rather than an occasional event. This approach is illustrated through an analysis of hiddenfigure items, and is shown to be feasible. Index terms: construct validity, generative modeling, isomorphic problems, item difficulty, spatial ability, validation.
\end{abstract}

Test validation has traditionally focused on an accounting of response consistency. Indeed, the most comprehensive form of test validationconstruct validity - has been described as implying "a joint convergent and discriminant strategy entailing both substantive coverage and response consistency in concert' (Messick, 1981, p. 575). There has been far less emphasis on an accounting of response difficulty (but see Campbell, 1961; Carroll, 1980; Davies \& Davies, 1965; Egan, 1979; Elithorn, Jones, Kerr, \& Lee, 1964; Tate, 1948; and Zimmerman, 1954).

Response consistency and response difficulty are not antithetical (e.g., the discussion of construct representation versus nomothetic span of Embretson, 1983). In fact, it could be argued that construct validity requires an accounting of

APPLIED PSYCHOLOGICAL MEASUREMENT

Vol. 15, No. 2, June 1991, pp. I29-137

(c) Copyright 1991 Applied Psychological Measurement inc. 0146-6216/91/020129-09\$1.70 response difficulty, in addition to requiring an accounting of substantive coverage and response consistency. Knowledge of the latent structure of a test (e.g., its factorial structure or its fit to a particular item response model) is clearly essential to an interpretation of test scores, but it is not sufficient. An accounting of response difficulty would clearly enhance the validation status of a test, because a model incorporating the mental structures and processes needed to solve the item would be required. If this model has been previously and independently validated, the validation status of the test clearly will be enhanced.

Not only are accounting of response difficulty and consistency not antithetical, but they entail parallel considerations. Within the responseconsistency tradition, for example, the extent to which covariation is accounted for by theoryrelevant and theory-irrelevant (e.g., method) variables is often the basic data from which validity is assessed (e.g., Campbell \& Fiske, 1959). Within the response-difficulty framework, the contributions of theory-relevant and theory-irrelevant processes to difficulty also can be addressed.

For example, clues that may have been inadvertently included in a test could result in some examinees not responding in accordance with their ability. Within the response-consistency framework this is reflected, in turn, in the itemresponse model's lack of fit. Under the same circumstances, within the response-difficulty framework examinees are viewed as not responding in accordance with the mental model postulated for 
a specific item. This response behavior is manifested as a discrepancy between the estimated difficulty of the item based on some itemresponse model, and the expected difficulty given the mental-process model for that item.

Discrepancies between difficulty estimates are well entrenched in psychometrics (e.g., Linn \& Harnisch, 1981). For example, discrepancies in the difficulty estimates from different groupsdifferential item performance-are often the focus of analysis. What may be new is that here one of the estimates is based on a substantive model of the mental effort required by an item.

An emphasis on accounting for response consistency is compatible with the latent-trait approach to individual differences. Lord (1980) included both factor analysis and item response theory (IRT) in this approach. Although an accounting of response difficulty also fits well within IRT, it requires inspiration from cognitive science in order to formulate mental models of the item solution process. Consider a test in which some item response model fits perfectly, and for which correlational analysis has established that it is a "verbal" test. It is tempting to stop there and argue that the test has been validated; indeed, many validation efforts do stop at this point.

There is more to explain, however: The items in the test differ in difficulty-some are very easy, and others very difficult. This variation presents no major problem, because every item response model includes a difficulty parameter. Yet estimating the difficulties is not the same as explaining them. Thus, when it is time to create a new form of the test, there is no method to predict the psychometric characteristics of an item nor to construct an item with known psychometric properties. The standard procedure followed by major testing organizations is to write many items and pretest them, with the hope that enough items will survive the process and a new form of the test that resembles the previous form can then be constructed. This procedure is effective, but it also underscores the fact that knowledge about the test is far from complete.
A thorough understanding of what a test measures can be demonstrated if test forms that are empirically parallel can be constructed on an a priori basis-that is, without resorting to items that already have been calibrated on a response model.

The objective of this research was to illustrate an approach to test modeling that encompasses both response consistency and response difficulty. This approach is termed generative for two reasons. It is generative in the usual dictionary sense of the word (i.e., "having the power of generating, originating, producing, or reproducing;" Webster's Ninth New Collegiate Dictionary, 1986, p. 912)-in this case, of items with known psychometric characteristics. But the approach may also be interpreted more broadly, as in Chomskyan linguistics (Chomsky, 1965), in which a generative grammar is defined as being capable of assigning a description to every sentence in the language, and also being capable of generating all the sentences in the language.

Generative psychometrics, then, involves a "grammar" capable of (1) assigning a psychometric description to every item in the universe of items, and (2) generating all the items in the universe of items. Some of these ideas are implicit in certain item-generation schemes (e.g., Bormuth, 1970; Roid \& Haladyna, 1982). However, the emphasis of these schemes is completely on generating-rather than assigning-a description with psychometric utility to the generated items. Therefore, these approaches are not generative. They cannot be generative in one sense, because the assignment of a psychometric description to an item requires an understanding of the underlying response process, which is outside the scope of approaches that are inspired by behavioristic principles. By contrast, a generative approach becomes feasible as a result of efforts to understand individual differences from the perspective of cognitive psychology. Indeed, Pellegrino (1988) noted that "cognitive scientists have been engaged wittingly and unwittingly in a process of construct validation" (p. 52).

Nothing in the above definition dictates what 
type of "description" should be attached to an item-other than its psychometric utility. In the context of ability testing, it would be natural to assign a description with respect to an itemresponse model, or to a response-time distribution. In the context of diagnostic testing, the description could be with respect to a set of misconceptions (Bejar, 1984; Brown \& Burton, 1978; Burton, 1982).

\section{Empirical Example}

\section{Method}

The concept of generative modeling is illustrated through an analysis of hidden-figure items. Figure 1 shows two sample items. The task of the examinee is to determine whether the smaller (or target) figure is embedded in the larger figure. Figure la is a "true" item because the figure on the right can be found within the larger figure; Figure $1 \mathrm{~b}$ is a "false" item. This item type has been used extensively in field dependenceindependence work; as a result, there is ample literature on correlating performance on hiddenfigure tests with personality variables (e.g., Witkin, Goodenough, \& Oltman, 1979). Unfortunately, nothing in that literature can be used as a means of constructing the procedure through

Figure 1

Sample Hidden-Figure Items

a. A True Item
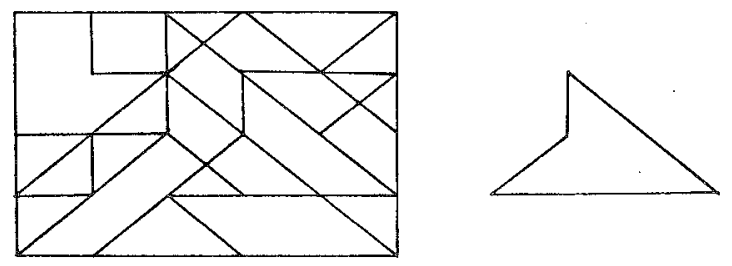

b. A False Item

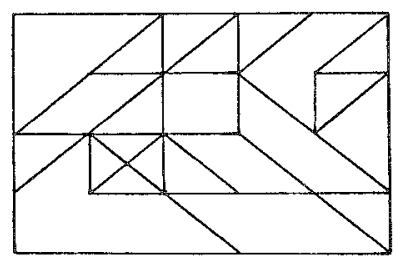

which items can be generated and a psychometric description can be assigned. The lack of such knowledge underscores the actuarial rather than the scientific nature of test development practices.

The approach selected to model performance on this item type was inspired by artificial intelligence research in vision (Mayhew \& Frisby, 1984), and it is based on a pattern-recognition algorithm called the Hough transform. This process is illustrated in Figure 2, which shows items of apparently increasing difficulty. The smaller figure in Figure 2 is the same for all the items, and consists of seven segments (the top line is two segments long). (All the target figures used here had seven segments.)

The matrix to the right of each item was computed by placing the smaller figure at each node of an invisible grid-the units of which were one segment-and counting the segments in the smaller figure that coincided with the segments in the larger figure. Each segment arbitrarily counted as 2. Thus, "14" indicated that the smaller figure was embedded in the larger figure, and a lesser number indicated that not all of the segments coincided.

The easiest item (Figure 2a) yields a matrix of matches with a 14 surrounded by $2 \mathrm{~s}$ and $4 \mathrm{~s}$. The most difficult item (Figure 2c), however, has several 12 s surrounding the 14 (i.e., there were many target-like figures surrounding the target figure, and it therefore became more difficult to disembed the target figure). When the target figure does not exist in the larger figure for false items, a similar analysis applies (i.e., many $12 \mathrm{~s}$ in close proximity confuse the examinee into "seeing" the target when it does not exist).

A full generative approach would link the matrix of segment matches to psychometric difficulty in such a way that items of a range of difficulty can be generated (Bejar, 1990b). A more modest objective, which may be necessary when such a complete link is not feasible, is to generate isomorphic items, or "clones" (i.e., in this case, to generate items with the same matrix of matches but different visual realizations). This was the approach taken here. Eight such pairs of 
Figure 2

Hidden-Figure Items of Increasing Complexity

a. Easy Item
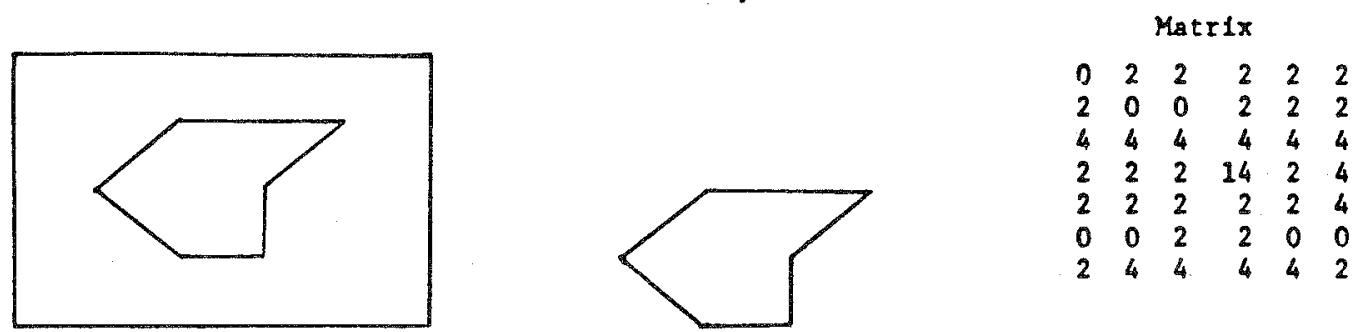

b. Moderately Difficult Item
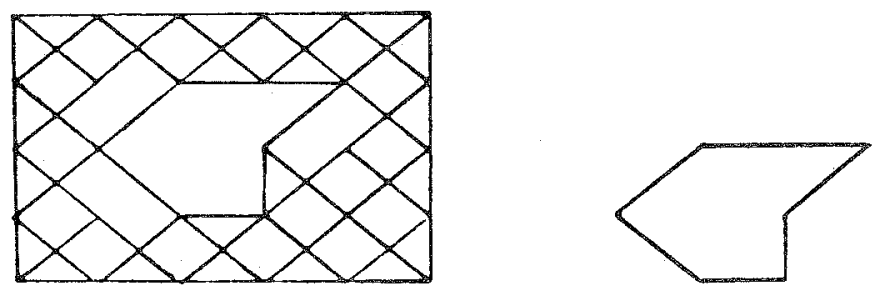

c. Difficult Item

\section{Matrix}

$\begin{array}{rrrrrrr}0 & 2 & 2 & 2 & 2 & 2 & 0 \\ 2 & 0 & 2 & 4 & 4 & 4 & 2 \\ 6 & 6 & 10 & 8 & 8 & 6 & 4 \\ 4 & 2 & 6 & 14 & 4 & 6 & 4 \\ 4 & 2 & 6 & 6 & 6 & 8 & 4 \\ 2 & 2 & 6 & 6 & 4 & 2 & 2 \\ 2 & 4 & 4 & 4 & 4 & 2 & 0\end{array}$
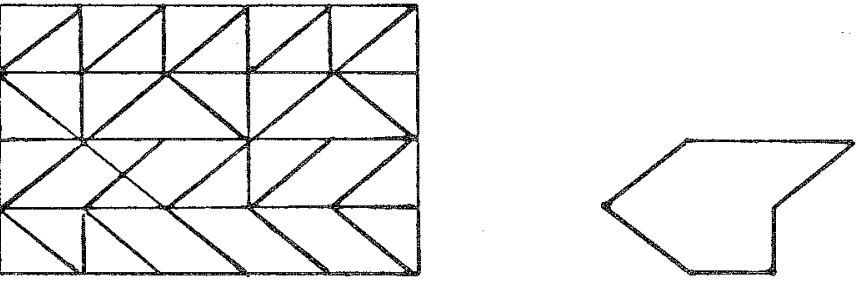

\begin{tabular}{rrrrrrr}
\multicolumn{8}{c}{ Matrix } \\
0 & 2 & 2 & 2 & 2 & 2 & 0 \\
2 & 4 & 4 & 4 & 4 & 4 & 0 \\
6 & 10 & 12 & 12 & 12 & 8 & 4 \\
4 & 8 & 6 & 14 & 6 & 8 & 0 \\
6 & 10 & 12 & 12 & 12 & 10 & 4 \\
2 & 4 & 4 & 4 & 4 & 2 & 0 \\
2 & 4 & 4 & 4 & 4 & 2 & 0
\end{tabular}

isomporphs were generated with a computer program. (A discussion of the program is beyond the scope of this report; see Ronse \& Devijver, 1984, for a discussion of a general program that uses a similar but far more general approach to the detection of embedded subfigures.) The generation component in the program used here, although not trivial, was simply an efficient search algorithm.

The item-generation algorithm used a target pattern and the matrix of matches described above as input and attempted to create a large pattern that yields the same matrix of matches. The generation process was simplified by the fact that segments were present and were removed until the algorithm produced a matrix that equaled the input matrix; the process was, therefore, recursive. If at some point the algorithm failed, it could backtrack as necessary.

\section{Items}

Eight items were selected from the The Kit of Cognitive Reference Factors (Ekstrom, French, \& Harman, 1976) as the generating items. The underlying matrix for each of these was computed. The resulting matrix was then used to generate eight pairs of isomorphs. The eight generating items, the eight pairs of isomorphs, and the in- 
structions for the items appear in Appendix A of Bejar and Yocom (1986).

The items were assembled into two forms: the first eight items were common to both forms A and $\mathrm{B}$ and consisted of the eight generating items; the last eight items consisted of set $\mathrm{A}$ of isomorphs for form A and set B of isomorphs for form $B$. The items were positioned in the two forms in such a way that corresponding isomorphs occupied the same position. Forms A and $\mathrm{B}$ were installed on an inexpensive graphics microcomputer (a Radio Shack Color Computer) with graphic resolution of $256 \times 192$. A color monitor (Amdek Color I) was used to display the items. Examinees responded by a means of a joystick (Radio Shack No. 26-3012). They were instructed to move the joystick forward if they thought the item was true and backward if they thought the item was false. Examinees' reaction times were recorded with a resolution of $1 / 60$ th of a second; response time was the elapsed time until a response was given, regardless of whether it was correct or incorrect. Examinees were informed if they were correct or incorrect after responding to each item.

\section{Examinees}

Sixty high school students participated, with approximately equal numbers of males and females. The data were not edited in any way prior to the analysis presented below. Form A was administered to 29 students, and Form B to 31 students.

\section{Results}

The validity of this approach to psychometric modeling was explored by examining the relationship between difficulty estimates for groups $A$ and $B$ on the generating items as well as on the isomorphs. To the extent that the approach is valid, it was expected that the difficulty estimates would not only be linearly related, but would fall along a line with a slope of 1 . An itemby-item analysis of the response-time distribution was also examined.

Item difficulties. Difficulty was estimated by the formula $\Delta=\log [p /(1-p)]$. Larger values of $\Delta$ are associated with more difficult items. Some of the statistical properties of $\Delta$ have been discussed recently by Holland and Thayer (1985). As can be seen in Figure 3a, the estimated difficulties tend to fall along a diagonal with a slope of 1.0, although the correlation between difficulty estimates was .41 . Figure $3 b$ shows the relationship between difficulty estimates for groups $\mathrm{A}$ and $\mathrm{B}$ responding to a different set of isomorphs. The relationship is strong $(r=.74)$, but more importantly, the estimates tend to fall along a diagonal line with slope of 1.0 .

A comparison of Figures $3 a$ and $3 b$ shows that a significant amount of change seems to have taken place within very few items. The median $\Delta$ for the generating items was approximately .5,

Figure 3

Relationship Between Estimates for Groups A and B a. Generating Items

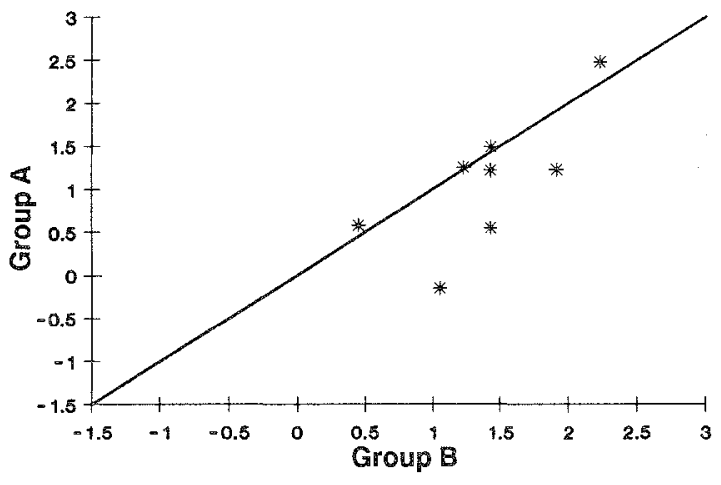

b. Pairs of Isomorphs From a Common Generating Item

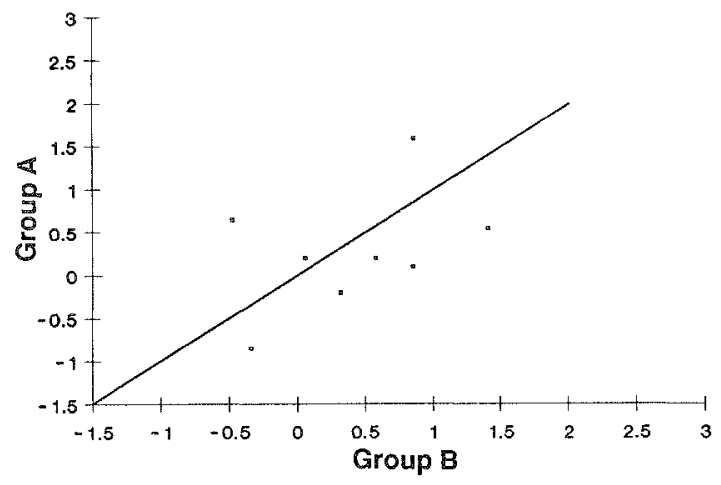


whereas it was 1.5 for the isomorphs, which were always administered subsequent to the generating items. In order to interpret this effect as learning rather than practice, a more complex design is needed.

Response times. Response time is more informative than difficulty in terms of whether or not the same psychological processes are involved in responding to items. Because groups A and B were randomly equivalent, it was expected that the cumulative response time distribution would be very similar for the two groups when responding to the generating item or the isomorphs. Figure 4a shows a typical cumulative response time distribution for groups A and B responding to the same generating item. As can be seen, the two distributions are very similar. Figure $4 \mathrm{~b}$

Figure 4

Typical Cumulative Response Time Distributions

a. Generating Item

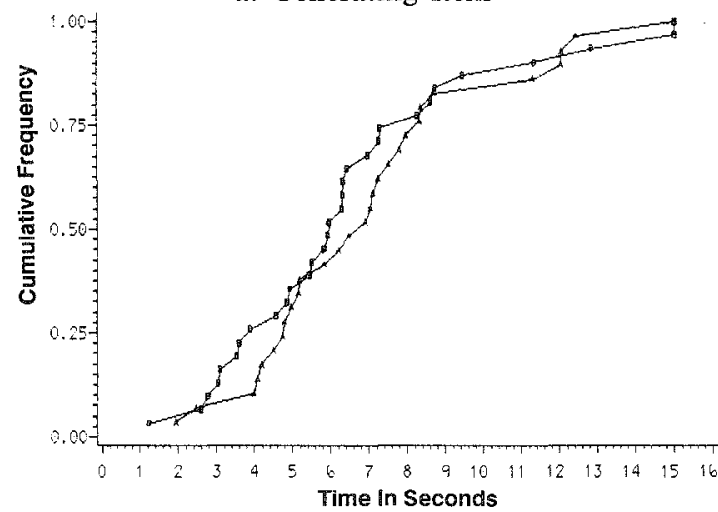

b. Two Isomorphs From the Same Generating Items

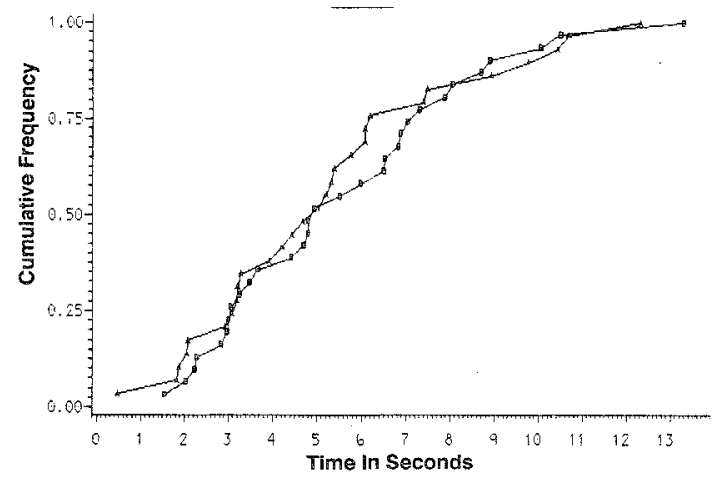

shows the corresponding distributions for responses for groups $\mathrm{A}$ and $\mathrm{B}$ responding to different isomorphs of the same generating item; these distributions are also very similar. (Due to space limitations, only a sample of the results is presented here; for complete results, see Bejar \& Yocom, 1986.) In general, the comparison of response time distributions suggested the effectiveness of the procedure in generating isomorphs. However, there were noticeable differences between generating items and isomorph; this result is discussed below.

\section{Discussion}

A generative approach to psychometric modeling incorporates response modeling, item development, and validation in a coherent and cohesive package. Response modeling and item development become a single process once the item generation algorithm for the item type in question is successfully operationalized.

As is true of other psychometric models, the possibility of misspecification exists. Just as a one-parameter logistic model, which is often used in psychometric work, may not adequately describe responses to multiple-choice items, the algorithm for a particular item type may not adequately generate isomorphic items. Thus, the validation phase is actually an integral part of the generative approach. Validity is built into test scores by creating items according to a performance model and then verifying that the predictions of the performance model are fulfilled, as manifested in psychometric parameters.

These findings serve primarily to illustrate the processes involved in the application of generative psychometrics because a psychological model for responding to hidden-figure items was not explicitly considered. However, the approach used to characterize the items was one in which the items would seem to be compatible with a template-matching approach. Although template matching as a theory of object recognition is not very tenable (e.g., Pinker, 1984), it does not seem unreasonable as the basic mechanism for disembedding a smaller figure from a larger one. 
Thus, performance on both true and false items like those used here appears to be controlled by the position and magnitude of the segment matches in the matrix: the more entries for true items that approach 14 (in the immediate neighborhood of the target), the longer it takes to arrive at a decision. Similarly, the number and distribution of counts below 14 for false items would seem to control performance. This is very consistent with recent accounts of visual search efficiency proposed by Duncan and Humphreys (1989).

The computational flavor of this description is certainly in line with information-processing cognitive psychology, but it seems to contradict Gestalt psychology, which would claim that perception cannot be understood simply as the sum of its parts. Some evidence in support of this claim is suggested by the difference in difficulty between generating items and their corresponding isomorphs. Although the isomorphs appeared last in the test here, it is not likely that their lower difficulty is only a position effect.

An alternative explanation is suggested by an examination of the generating items and their isomorphs, which demonstrates that symmetry is a global feature of the generating item that is not preserved by the generation algorithm. Symmetry is known to play an important role in the recall, recognition, and discrimination of figures (Adams, Fitts, Rappaport, \& Weinstein, 1954; Attneave, 1955; Chipman 1977; Royer, 1981; Soltz \& Wertheimer, 1959). It is thus possible that the higher difficulty of the generating items is due to perceptual features that are beyond the grasp of the generation algorithm. Further research should therefore assess the impact of these global features in the context of the hidden-figure item type. If they are found to be important, the feasibility of generative psychometrics for this item type will rest on the possibility of incorporating those global features in both the description and generation phases of a generative algorithm.

Although generative modeling was illustrated here in the context of a specific item type, it is a general approach for incorporating substantive considerations into test development and psychometric modeling independent from specific statistical details of the response model. In addition, a generative approach should be equally feasible within such frameworks as IRT or generalizability theory. This approach is also general in terms of domains of application. Although the focus of this analysis was on the hidden-figure item type, generative modeling is also possible in other domains, including writing assessment (e.g., Bejar, 1988), analogical reasoning (Bejar, Chaffin, \& Embretson, 1991), and presumably any domain in which a theory of performance is available or can be developed (Bejar, 1990a).

In principle, generative modeling may be an economical approach to adaptive testing in which considerable resources must be invested in the calibration and maintenance of the item bank (Stocking, 1988). The potential economy of generative modeling follows from the fact that item generation algorithms are calibrated, rather than items. Efficiency considerations aside, the real contributions of a generative approach lies in the demands that it imposes on psychometricians who have permitted item writers to take charge of the creation of items, thus reserving for themselves the task of estimating the parameters of some response model. A generative approach should therefore bring the item writer, the psychometrician, and the cognitive scientist closer together. The validity of scores from tests developed in this manner is likely to be greater than that of conventionally developed tests.

\section{References}

Adams, O. S., Fitts, P. M., Rappaport, M., \& Weinstein, M. (1954). Relations among some measures of pattern discriminability. Journal of Experimental Psychology, 48, 81-88.

Attneave, F. (1955). Symmetry, information, and memory for patterns. American Journal of Psychology, 68, 209-222.

Bejar, I. I. (1984). Educational diagnostic assessment. Journal of Educational Measurement, 21, 175-189.

Bejar, I. I. (1988). A sentence-based automated approach to the assessment of writing: A feasibility study. Machine-Mediated Learning, 2, 321-332.

Bejar, I. I. (1990a). A generative analysis of a three- 
dimensional spatial task. Applied Psychological Measurement, 14, 237-245.

Bejar, I. I. (1990b). A generative approach to psychological and educational measurement. In $\mathrm{N}$. Frederiksen, R. J. Mislevy, \& I. I. Bejar, (Eds.), Test theory for a new generation of tests. Hillsdale $\mathrm{NJ}$ : Erlbaum.

Bejar, I. I., Chaffin, R., \& Embretson, S. (1991). Cognitive and psychometric analysis of analogical problem solving. New York: Springer-Verlag.

Bejar, I. I., \& Yocom, P. (1986). A generative approach to the development of hidden-figure items (Report No. RR-86-20-ONR). Princeton NJ: Educational Testing Service.

Bormuth, J. R. (1970). On the theory of achievement test items. Chicago IL: University of Chicago Press.

Brown, J. S., \& Burton, R. R. (1978). Diagnostic models for procedural bugs in basic mathematical skills. Cognitive Science, 2, 155-192.

Burton, R. R. (1982). Diagnosing bugs in simple procedural skills. In D. Sleeman \& J. S. Brown, (Eds.), Intelligent tutoring systems. New York: Academic Press.

Campbell, A. C. (1961). Some determinants of the difficulty of non-verbal classification items. Educational and Psychological Measurement, 21, 899-913.

Campbell, D. T., \& Fiske, D. W. (1959). Convergent and discriminant validation by the multitraitmultimethod matrix. Psychological Bulletin, 56, 81-105.

Carroll, J. B. (1980). Measurement of abilities constructs. In Construct validity in psychological measurement: Proceedings of a colloquium on theory and application in education and employment. Princeton NJ: Educational Testing Service.

Chipman, S. (1977). Complexity and structure in visual patterns. Journal of Experimental Psychology: General, 106, 269-301.

Chomsky, N. (1965.) Aspects of the theory of syntax. Cambridge MA: MIT Press.

Davies, A. D. M., \& Davies, M. G. (1965). The difficulty and graded scoring of Elithorn's perceptual maze test. British Journal of Psychology, 14, 295-302.

Duncan, J., \& Humphreys, G. W. (1989). Visual search and stimulus similarity. Psychological Review, 96, 433-458.

Egan, D. E. (1979). Testing based on understanding: Implications from studies of spatial ability. Intelligence, 3, 1-15.

Ekstrom, R., French, J., \& Harman, H. (1976). Kit of factor-referenced cognitive tests. Princeton NJ: Educational Testing Service.

Elithorn, A., Jones, D., Kerr, M., \& Lee, D. (1964). The effects of the variation of two physical parameters on empirical difficulty in a perceptual maze test. British Journal of Psychology, 55, 31-37.

Embretson, S. E. (1983). Construct validity: Construct representation versus nomothetic span. Psychological Bulletin, 93, 175-197.

Holland, P. W., \& Thayer, D. T. (1985). An alternate definition of the ETS delta scale of item difficulty (Technical Rep. 85-86). Princeton NJ: Educational Testing Service.

Linn, R. L., \& Harnisch, D. L. (1981). Interaction between item content and group membership on achievement test items. Journal of Educational Measurement, 18, 109-118.

Lord, F. M. (1980). Applications of item response theory to practical testing problems. Hillsdale $\mathrm{NJ}$ : Erlbaum.

Mayhew, J., \& Frisby, J. (1984). Computer vision. In T. O'Shea and M. Eisenstadt (Eds.), Artificial intelligence: Tools, techniques, and applications. New York: Harper \& Row.

Messick, S. (1981). Constructs and their vicissitudes in educational and psychological measurement. $P s y-$ chological Bulletin, 89, 575-588.

Pellegrino, J. W. (1988). Mental models and mental tests. In H. Wainer \& H. I. Braun (Eds.), Test validity. Hillsdale NJ: Erlbaum.

Pinker, S. (1984). Visual cognition: An introduction. In S. Pinker (Ed.), Visual cognition. Cambridge MA: MIT Press.

Roid, G. H., \& Haladyna, T. M. (1982). A technology for item writing. New York: Academic Press.

Ronse, C., \& Devijver, P. A. (1984). Connected components in binary images: The detection problem. New York: Wiley.

Royer, F. L. (1981). Detecting symmetry. Journal of Experimental Psychology: Human Perception and Performance, 7, 1186-1210.

Soltz, D. F., \& Wertheimer, M. (1959). Retention of "good" and "bad" figures. American Journal of Psychology, 72, 450-452.

Stocking, M. (1988). Some considerations in maintaining adaptive test item pools (Report No. RR-83-33-ONR). Princeton NJ: Educational Testing Service.

Tate, M. W. (1948). Individual differences in speed of response in mental test materials of varying degrees of difficulty. Educational and Psychological Measurement, 8, 353-374.

Webster's Ninth New Collegiate Dictionary. (1986). Springfield MA: Merriam-Webster.

Witkin, H. A., Goodenough, D. R., \& Oltman, P. K. (1979). Psychological differentiation: Current status. Journal of Personality and Social Psychology, 37, $1127-1145$.

Zimmerman, W. S. (1954). The influence of item com- 
plexity upon the factor composition of a spatial visualization test. Educational and Psychological Measurement, 14, 106-119.

\section{Acknowledgments}

This research was sponsored by the Personnel and Training Research Programs, Psychological Sciences Division, Office of Naval Research, under Contract No. N00014-83-C-0761, NR 150-531. The authors thank Lisa Jansen for assisting with data collection and database maintenance, Ka-Ling Chan for the statistical analysis, and Jessie Cryer for typing the manuscript. Thanks also to Susan Embretson and Philip Oltman for valuable comments, and to Miguel A. Garcia-Perez for a number of insightful comments. Finally, the author thanks two anonymous reviewers for their helpful comments.

\section{Author's Address}

Send requests for reprints and further information to Isaac I. Bejar, Educational Testing Service, Princeton NJ 08541, U.S.A. 